\title{
Synchronous Brightenings Of Microwave Emission Of Solar Active Regions According To The RATAN-600 Spectral Data
}

\author{
Olga A. Golubchina ${ }^{1}$, S. Kh. Tokhchukova ${ }^{2}$, V. M. Bogod ${ }^{1}$, \\ H. A. Garcia ${ }^{3}$, and V. I. Garaimov ${ }^{1}$ \\ ${ }^{1}$ St. Petersburg Branch of the Special Astrophysical Observatory 196140, St. Petersburg, \\ Russia email: oag@OG4466.spb.edu \\ ${ }^{2}$ Main (Pulkovo) Astronomical Observatory, 196140, St. Petersburg, Russia \\ ${ }^{3}$ NOAA Space Environment Center, Boulder, USA
}

This paper is devoted to the investigation of the synchronous enhancements of the solar source fluxes according to the RATAN-600 Southern Sector observations at the $1.92 \mathrm{~cm}, 2.24 \mathrm{~cm}, 2.74 \mathrm{~cm}$, and $3.21 \mathrm{~cm}$ radio waves. The observations were carried out on 11.09.01 from 8:30 UT to 10:30 UT every $8 \mathrm{~min}$. We use the term "synchronous bursts" or "synchronous brightenings" instead of the "sympathetic bursts" as the time resolution of the RATAN - 600 is not high. The solar source brightenings are synchronous if they are registered in $2-2.5 \mathrm{~min}$ time lag, i.e. in the time interval of passage of the Sun through the central part of the main lobe of the antenna power pattern. The high flux sensitivity of the radio telescope - radiometer system was 0.005 s.f.u. The solar sources were identified with the active regions: AR 9610, AR 9608, AR 9606, AR 9616, AR 9617, which widely separated from each other (up to $10^{6} \mathrm{~km}$ ). According to our observations, the synchronous enhancements were observed at the moments of the soft $\mathrm{X}$ ray brightenings and $\mathrm{X}$ ray bursts (Figure 1a). The temporal variations of the relative fluxes of the solar sources $F / F_{0}$ were compared to the $X$-ray (GOES 8,10; $H_{\alpha}$ - flares and to the radio bursts at $9100 \mathrm{MHz}, F_{0}$ - the radio emission flux from the "quiet" Sun, $F$ the radio emission flux from the solar source). It turned out that the interrelationships of the active regions AR 9610, AR 9608, AR 9606 were the most interesting. It was registered 4 synchronous brightenings of solar sources AR 9610, AR 9608 at the moment of the observations: 8:38 UT, 9:02 UT, 9:50 UT, 10:30 UT. We recorded synchronous brightenings of solar sources at $1.92 \mathrm{~cm}, 2.24 \mathrm{~cm}, 2.74 \mathrm{~cm}$, and $3.21 \mathrm{~cm}$ radio waves: at $\lambda=2.24 \mathrm{~cm}-\Delta(F)=(0.24 \div 0.88)$ s.f.u. and at $\lambda=3.21 \mathrm{~cm}-\Delta(F)=(0.21 \div 0.94)$ s.f.u. The detailed halo solar source is the magnetosphere, which was identified with all extensive AR 9608 region excepting the primary the most strong solar source of the active region radio emission. The angular size of halo is closed to the angular size of AR 9608 $\left(7.7^{\prime}\right)$. The comparison of the brightening onset of the AR 9610, AR 9608 interacting active regions in the time interval (9:26 - 9:58 UT) and the discovered forestalling of the halo flux quick intensification at $\lambda=2.24 \mathrm{~cm}$ and $\lambda=3.21 \mathrm{~cm}$ pointed to the active process start in the AR 9608 solar source at $\lambda=3.21 \mathrm{~cm}$. As it was shown, the dynamics of the time intensity variation of solar sources at $\lambda=3.21 \mathrm{~cm}, \lambda=2.74 \mathrm{~cm}$ is more complicated. It is demonstrated by against of the synchronous of the moments of the AR 9610 relative flux maxima with the $\lambda$ increase (Figure 1b).

It was obtained the evaluation of the disturbing agent velocity from $V=0.3 \times 10^{3}$ $\mathrm{km} / \mathrm{s}$ to $V \geqslant 10^{4} \mathrm{~km} / \mathrm{s}$. The effect of the high energy electrons predominates at short wave lengths $(\lambda=1.92 \mathrm{~cm}, 2.24 \mathrm{~cm})$. These high energy electrons $\left(V \geqslant 10^{4} \mathrm{~km} / \mathrm{s}\right)$ escaped from the initial burst site and traveled to the site of the second burst along 


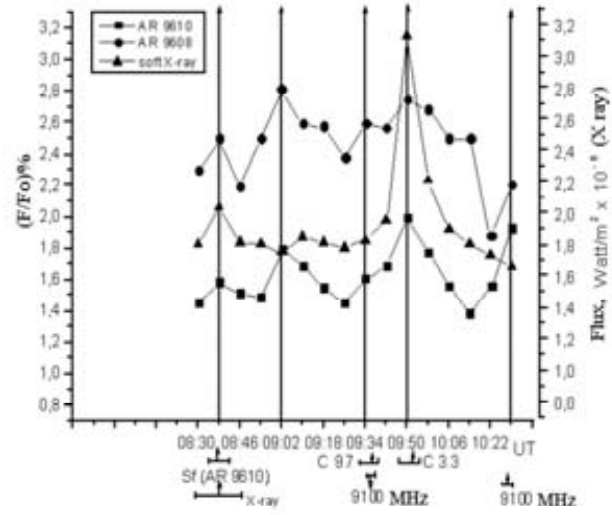

a)

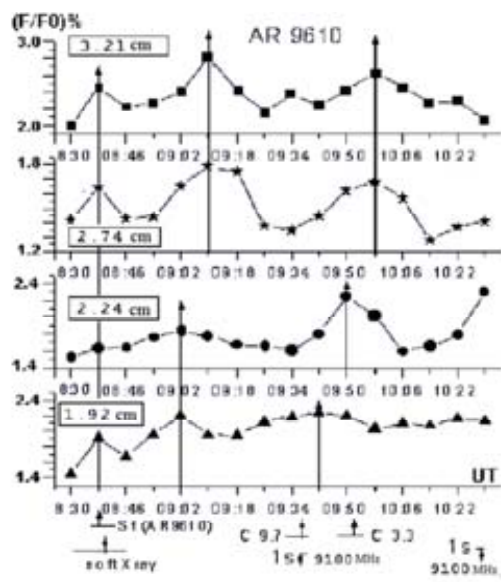

b)

Figure 1. a)The relative flux time variations of solar source radiations AR 9610, AR 9608 at $\lambda$ $=2.24 \mathrm{~cm}$ and the $\mathrm{X}$ ray time variation. Arrows up: the time intervals of the $\mathrm{H} \alpha-$ flare and of the $\mathrm{X}$ ray burst; arrows down: the time interval of the burst at $9100 \mathrm{MHz}$. b) The relative flux time variations of AR 9610 solar source radiation at $\lambda=1.92 \mathrm{~cm}, 2.24 \mathrm{~cm}, 2.74 \mathrm{~cm}, 3.21 \mathrm{~cm}$.

the magnetic loops connecting these AR 9608, AR 9610 active regions. The two trigger mechanisms are revealed at more long waves $(\lambda=2.74 \mathrm{~cm}, 3.21 \mathrm{~cm})$ : the effect of the wave fronts extended from the initial burst site $\left(V=(3-7) \times 10^{2} \mathrm{~km} / \mathrm{s}\right)$ after the rapid high energy electrons. They amplify the sympathetic bursts which aroused as a result of the high energy electrons effect earlier. The registered synchronous brightenings of the AR 9608, AR 9610 solar sources at $\lambda=1.92 \mathrm{~cm}, 2.24 \mathrm{~cm}$ point to the existence of the closed connections of these solar sources separated by a distance $\sim 2 \times 10^{5} \mathrm{~km}$. We obtained the significant linear correlation of the AR 9608, AR 9610 radio emission at $\lambda=1.92 \mathrm{~cm}, 2.24 \mathrm{~cm}(\rho=0.84-0.92, \mathrm{P}=0.04-0.01 ; \rho$ - the coefficient of the linear correlation, $\mathrm{P}$ - the significance level). The significant linear correlation $(\rho=0.65-0.84$, $\mathrm{P}=0.01-0.04$ ) for the AR 9608, AR 9606 radio emission was obtained at $\lambda=3.21 \mathrm{~cm}$. These active regions are $3.9 \times 10^{5} \mathrm{~km}$ apart. It is shown the connection of the AR 9608 , AR 9606 radio emission for the whole interval of our observation.

Thus, it was revealed the linear correlation of the large - scale time component of dynamic of the flux radio emission of the AR 9608, AR 9610, AR 9606 solar sources. That verifies the existence of the solar source connection during whole time interval of our observations with the RATAN - 600 .

\section{Conclusions}

The observations of the radio emission of the Sun with the radio telescope RATAN 600 at four cm - wave-lengths: $\lambda=1.92 \mathrm{~cm}, 2.24 \mathrm{~cm}, 2.74 \mathrm{~cm}, 3.21 \mathrm{~cm}$ for two hours on 11 September 2001 confirmed the earlier findings conclusion: the synchronous enhancements of the solar source fluxes and sympathetic bursts are not exotic phenomena. Besides, the results of the presented study pointed to the manifestation of the two different trigger mechanisms of the solar source synchronous brightenings at the narrow spectral range of the microwave emission: the effect of the high energy electrons traveling along the magnetic loops which connect the initial and the second burst sites $(\lambda=1.92 \mathrm{~cm}, 2.24 \mathrm{~cm})$ and the effect of the high energy electrons and then the effect of the wave fronts extended from the initial burst site $(2.74 \mathrm{~cm}, 3.21 \mathrm{~cm})$. 